\title{
LA DOMUS DI EUROPA A FORUM SEMPRONII
}

\author{
Filippo Venturini \\ venturini.filippo@libero.it \\ Parco ArCheologico di Forum Sempronil (Fossombrone, PU)
}

\begin{abstract}
The Domus of Europa has been under excavation since 2004. This particular building seems more like a labyrinth than like a Roman private house at first glance, due to its complex plan. The typical Roman domus is characterized by an axial and symmetrical disposition of the rooms, which seems to be completely missing in the Domus of Europa. This labyrinth like plan is due to the fact that this house is the result of the union of two different buildings, named respectively as "Building A" and "Building B". In the following pages, the life phase of these two buildings will be outlined into their junction during 150-200 A.D. until the destruction of the Domus of Europa due to fire, which has been agreed to have happened during the IV century A.D. After this tragic event, it is argued here that during the V Century A.D. a new building rose on top of the old one (referred to as "Building C").
\end{abstract}

La domus mutua il nome da un mosaico ivi rinvenuto, composto da tre pannelli figurati, in uno dei quali, quello centrale, è ritratta Europa sul Toro. L'edificio fu parzialmente scavato fra il 1878 e il 1880, per poi essere rinterrato (Fig. 1), nel 1926, sulla scorta delle piante e dei rilievi del ' 800 , venne nuovamente individuata la stanza con il mosaico, che fu di nuovo scavata e l'opera tessellata fu asportata, insieme ad un altro pavimento musivo geometrico, rinvenuto in una stanza vicina. Nel 2004, nell'ambito dell'attività di scavo svolta dall'Università degli Studi di Urbino, nel sito di Forum Sempronii, ricorrendo ai documenti risalenti alla fine del XIX secolo e a quelli del 1926, si decise di riaprire l'area, per uno studio approfondito di quella che sembrava essere una lussuosa domus privata. Alla campagna di scavo del 2004 seguì una pubblicazione dei primi risultati ${ }^{1}$, ma questa non poteva che essere una trattazione parziale con deduzioni dal valore provvisorio, visto che l'articolo in questione era incentrato soprattutto sui mosaici, l'unica parte scavata era quella già indagata nel XIX secolo.

Oggi è stata riportata alla luce unampia porzione della domus, comprese ampie parti scavate ex novo, tuttavia, a nord, ancora molte stanze giacciono sotto terra e in buona parte sono al di là dei limiti della concessione di scavo (Figg. 2-3).

Ledificio pone una serie piuttosto complessa di problemi, a molti dei quali non si è ancora riusciti a dare delle risposte. Ė di fondamentale importanza individuare le principali criticità del caso. Innanzi tutto si deve tenere ben presente che la domus non sorse su di unarea piana, 


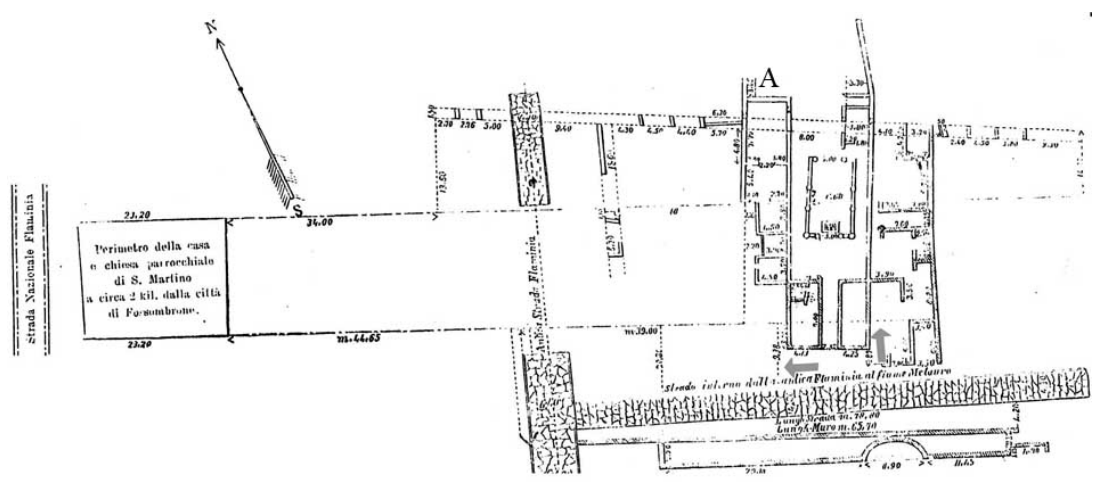

Fig. 1 - Rilievo del Vernarecci del 1880, ambienti dell'edificio A, a sud un peristilio pertinente ad un'altra domus.

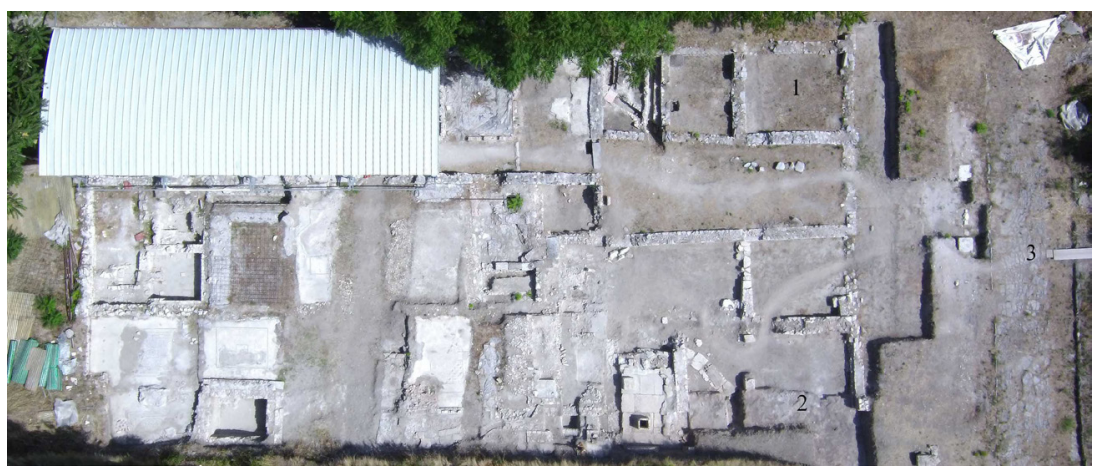

Fig. 2 - La Domus di Europa allo stato attuale: (1) Edificio A; (2) Edificio B; (3) Cardo.

ma lungo un pendio, attualmente tuttora percepibile, ma che in antico doveva essere decisamente più accentuato e che va crescendo in senso sud-nord, raggiungendo il culmine in corrispondenza del foro. Gli ambienti della domus più meridionali sono quelli con pavimenti alle quote più basse, mentre quelli più a nord li hanno alle quote più alte (Fig. 4), il fatto è chiaramente connesso con la particolare morfologia del terreno e dunque obbliga ad applicare con estrema cautela l'equazione: pavimento più alto = pavimento più tardo, seppur in alcuni casi anche questo sia un elemento da tenere in considerazione.

Basterà osservare la pianta dell'edificio (Figg. 2-3) per notare come questo abbia ben poco di canonico per una domus romana, ma sembri piuttosto un dedalo di stanze e corridoi, questa è la conseguenza del fatto che è la risultante dell'accorpamento di due fabbriche diverse, che abbiamo denominato: Edificio A e Edificio B, quest'ultimo non è stato completamente indagato e in buona parte giace al di là del limite dellarea di scavo. 


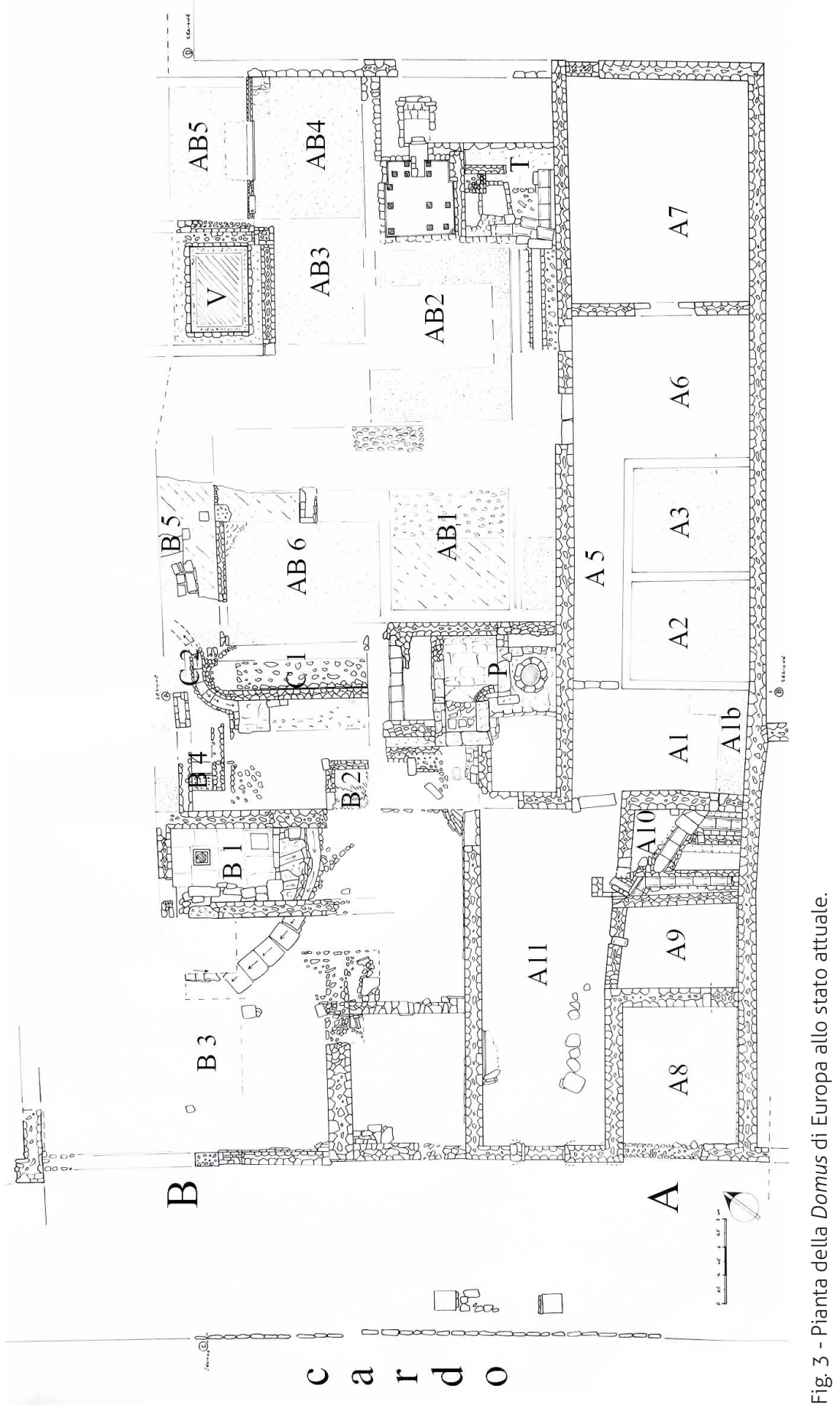



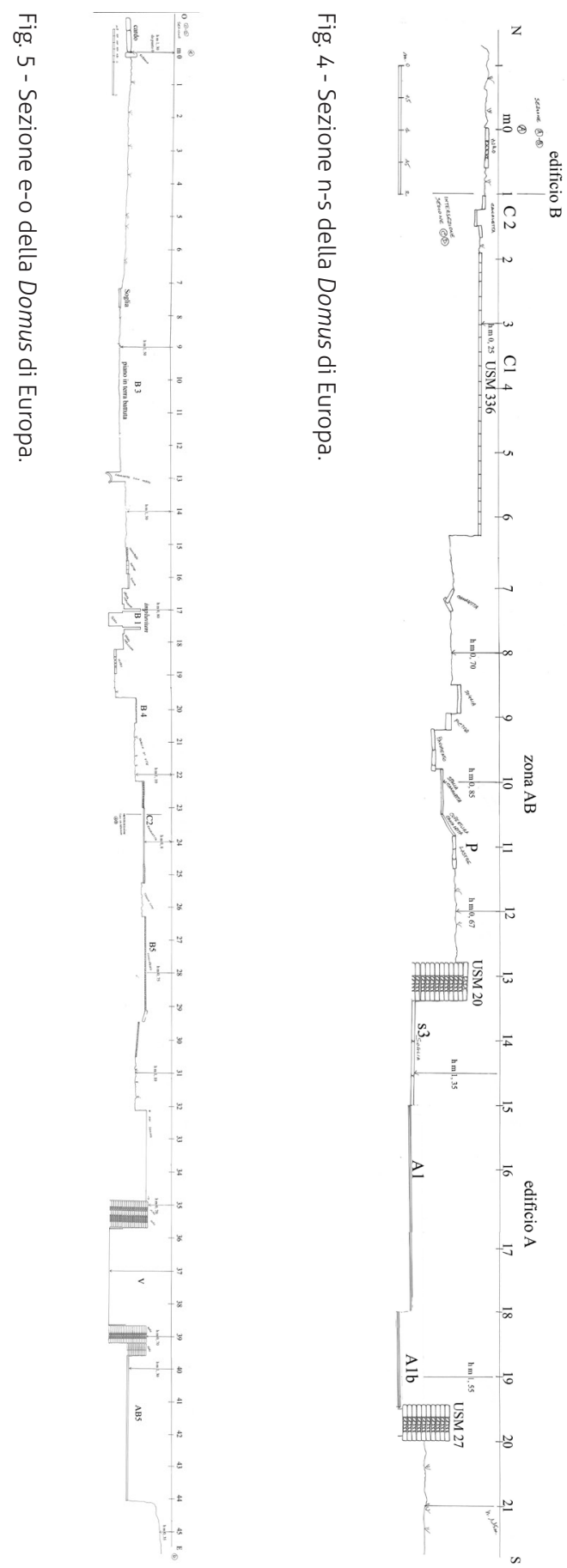


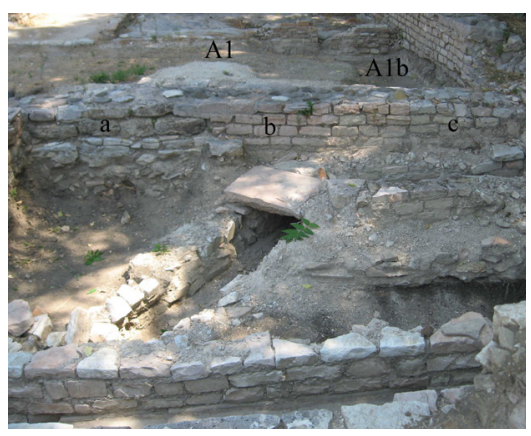

Fig. 6 - Edificio A, latrina. Si notano le diverse fasi: (a) Fase I; (b) Fase II; (c) Fase III, cioè tamponatura della porta.

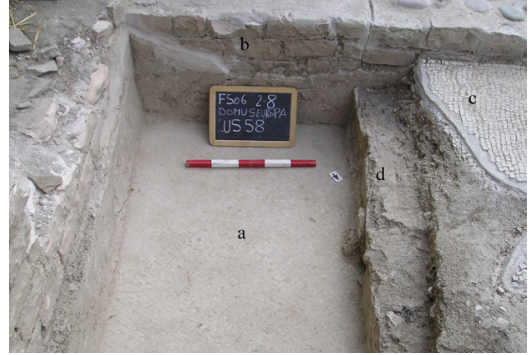

Fig. 7 - Edificio A, ambiente A 1, con le sue varie fasi:(a) livello pavimentale originario, poi trasformato in A 1b; (b) tamponatura della porta; (c) livello pavimentale della Fase IV; (d) muretto divisorio fra A 1 e A 1 b.

Il primo è quello più a sud ed è quello meglio conosciuto, dato che è stato completamente scavato, ma è quella parte della domus che fu già scavata dal Vernarecci alla fine del XIX secolo (Fig. 1). In questa sede verranno analizzati i due edifici A e B singolarmente, descrivendone l'evoluzione strutturale, giungendo fino all'epoca dell'unione, con la conseguente creazione della Domus di Europa, fino alla distruzione e all'ultima frequentazione dell'area, testimoniata da un edificio che in parte sembra sorgere sulle macerie della domus, in parte sembra riutilizzarne alcune stanze.

\section{EdIFICIO A}

\section{Fase I}

All'estremità ovest dell'edificio ci sono i resti di un muro evidentemente più antico di tutti gli altri, non è possibile dire con certezza, se testimoni la fase primigenia dell'edificio o sia pertinente ad unaltra struttura, poi obliterata o distrutta (Figg. 5-6).

\section{Fase II}

Il muro più antico viene inglobato in una struttura muraria nuova, con paramento in pietra della Cesana, questo nuovo muro si arrestava a sud, per la presenza di una porta, poi tamponata (Figg. 6-7). Il pavimento era a mosaico, quanto resta in loco è con tessere bianche, disposte in diagonale di $\mathrm{cm} 1$ in media di lato. Tuttavia non è chiaro di che tipo di edificio si tratti.

Elementi datanti: muratura in opera vittata ${ }^{2}$. Connessi con questa fase potrebbero essere i frammenti di intonaco dipinto che furono rimpiegati nella preparazione del pavimento delle terme della domus. 


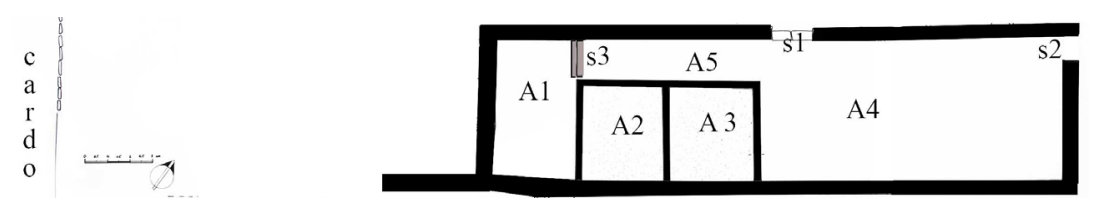

Fig. 8 - Edificio A: Fase III.

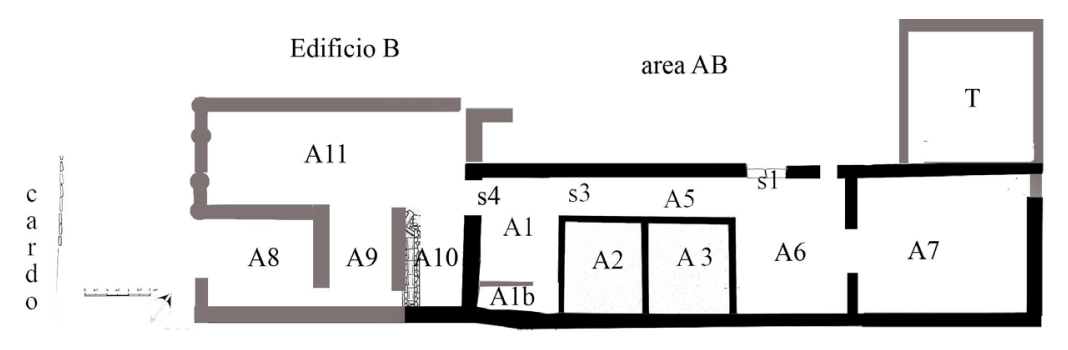

Fig. 9 - Edificio A: Fase IV.

Si tratta di frammenti di decorazione parietale attribuibili alla fine del I a.C., inizio del secolo successivo ${ }^{3}$. Datazione: prima metà del I d.C. ${ }^{4}$

\section{Fase III (Fig. 8)}

Vengono realizzati in due grandi muri a sud (USM 27) e a nord (USM 20), vengono realizzate le stanze A 1-5. In A1 resta il vecchio pavimento a mosaico, ad un livello più basso rispetto a quello degli altri ambienti, doveva quindi esserci un grandino fra A 1 e A 5 (s 3). In questa fase si chiude la porta nell'angolo sud ovest di A 1 (Fig. 7). A est di A 3 cè un ampio vano (A 4), con una porticina nell'angolo nord est (s 2). In A 7 sono stati trovati resti di incannucciato, spesso i vani dei piani superiori erano realizzati con muri costruiti con pani rettangolari di argilla, impastata con paglia e i solai tra un piano e l'altro erano formati da travi, chiuse da assiti, al di sotto di questa orditura era fissata un'incannucciatura rivestita di malta ${ }^{5}$. Si potrebbe quindi ipotizzare che attraverso la porta s 2, si accedesse ad una scala di legno, che avrebbe potuto condurre ad un piano superiore. All'estremità occidentale attualmente si nota una soglia in pietra (Fig. 10), ma data la quota è evidente che questa sia connessa con una fase ulteriore, in cui fu rialzato il livello pavimentale. In questo momento di vita della casa il muro doveva essere continuo, prova ne è il fatto che la soglia è stata evidentemente posta in opera tagliandolo. È dunque verosimile che l'ingresso dell'edificio fosse lungo il corridoio A 5 , più precisamente in corrispondenza di $s$, che

3 Graziani, Poloni, 2012, p.186-191.

4 Si riportano le datazioni iniziali di ogni fase.

5 Ortalli, 2000, p. 513. 


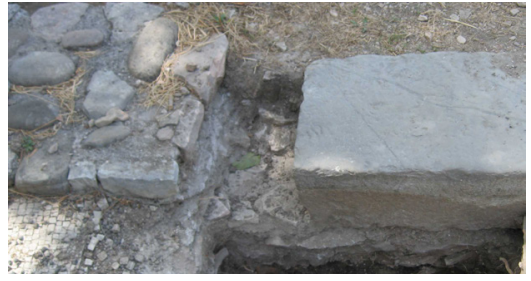

Fig. 10 - Edificio A, Fase IV, soglia s4, realizzata tagliando il muro più antico.

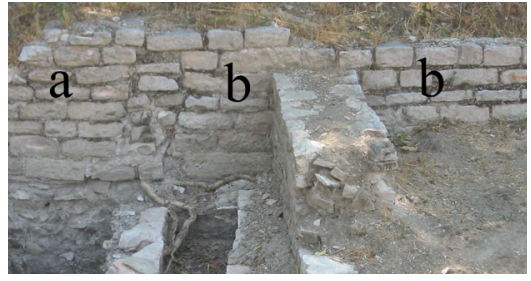

Fig. 11 - Edificio A, Fase IV (a) USM 27, alla quale viene addossato (b) USM 100.

poi sarà usata come soglia di passaggio all'altra parte delledificio, dopo l'accorpamento.

Elementi datanti: mosaici delle stanze A 2, A 3, soprattutto il secon$\mathrm{do}^{6}$; ceramica trovata nei saggi: sigillata italica. La pianta della casa trova un puntuale confronto con le "casette tipo" di Ostia, datate ad epoca Traianea $^{7}$. Datazione: fra fine I d.C. e inizio II d.C.

\section{Fase IV (Fig. 9)}

Viene realizzata la stanza di Europa (A 7) con l'aggiunta dei due setti murari occidentali, che delimitano l'ingresso al vano, questi sono appoggiati ai muri sud e nord e ne coprono anche l'intonaco di rivestimento. Di conseguenza viene creato anche il vano A 6. Viene chiusa s 2, si realizza l'ambiente termale (T) e anche l'ingresso a ovest (s 4) (Fig. 10), necessario giacché quello precedente (s 1) è diventato il punto di passaggio dall'Edificio A a quello B, ormai accorpati. Con l'apertura dell'ingresso s 4, si innalza il livello pavimentale in A 1 (Fig. 7), realizzando un rozzo mosaico in grosse tessere bianche, che riprende lo stile di quello che pavimentava A 5, del quale restano solo alcuni lacerti, ma che aveva uno strato di preparazione di ottima fattura, mentre il nuovo mosaico in A 1 poggia su strati preparatori di pessima consistenza, questa è una prova evidente, che nonostante la somiglianza delle tessere, i mosaici di A 5 e A 1 non siano coevi. Il precedente mosaico di A 1 non viene tuttavia completamente coperto, se ne lascia una parte, nell'angolo sud della stanza, creando una sorta di sgabuzzino (A1b) (Fig. 7), che a nord era delimitato da un muro divisorio di pessima fattura, del quale ne restano ancora le tracce. Il motivo della creazione di questo piccolo ambiente non è chiaro, ma visto che in questa fase viene chiusa s 2 , che verosimilmente conduceva alla scala per il piano superiore è possibile, che in questo piccolo ambiente si ponesse una scala lignea, del resto anche la forma dellambiente indirizzerebbe in questo senso ${ }^{8}$. 


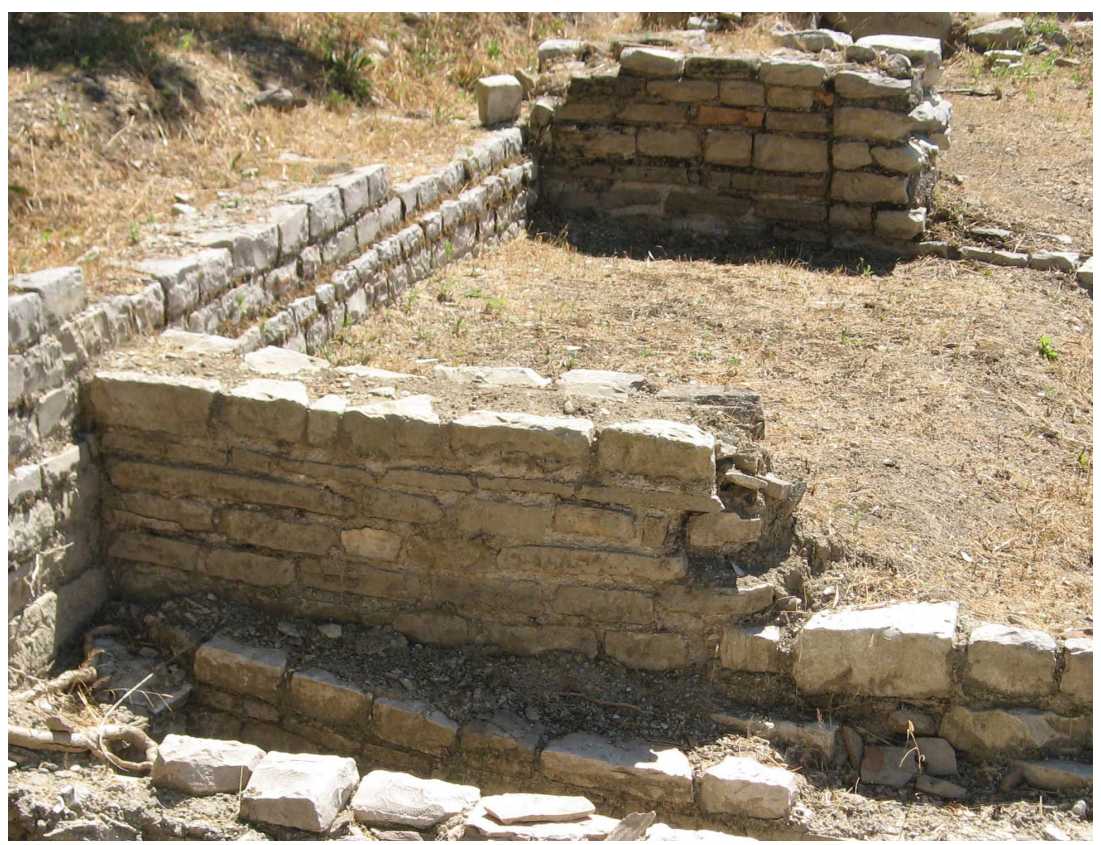

Fig. 12 - Edificio A, Fase V: tamponature delle porte di A 8 e A 9, con mura in corsi alternati di laterizio e pietra.

In questa fase a USM 27 si aggiunge un nuovo tratto (USM 100) (Fig. 11), si creano gli ambienti A 8, A 9, A 10 nonché l'ingresso monumentale, verso il cardo, con due colonne centrali e due semicolonne laterali (A 11).

Lambiente A 8 era aperto sul cardo, aveva poi una porta nell'angolo sud est che immetteva in A 9, da qui con una porta nell'angolo sud est si poteva passare in A 10, che era una latrina, con il solo canale occidentale e che aveva un ingresso anche a nord. A 8 e A 9 erano verosimilmente delle botteghe, così la latrina serviva sia queste che la domus, il che fa pensare che il proprietario fosse lo stesso.

Elementi datanti: mosaici'; pitture parietali: nella stanza AB 4 sono stati ritrovati in crollo numerosi frammenti della decorazione parietale, nella quale era ritratto il mito di Perseo ed Andromeda, scena che era immortalata fra riquadri di fasce multicolore, che rimandano chiaramente a esempi pittorici databili fra il 150 d.C. e l'inizio del secolo successivo. Come confronti si possono citare: l'Insula di Giove e Ganimede, tablino 14 fine del II d.C. ${ }^{10}$, ma anche la Caupona del Pavone, Ostia, ambiente IX riquadri con fasce multicolori età severiana ${ }^{11}$. Datazione: 150-200 d.C.

9 Venturini, 2007, p. 64-90.

10 Falzone, 2004, p. 61-74; Clarke, 1991, p. 320-339.

11 FALzone, 2004, p. 178-181. 

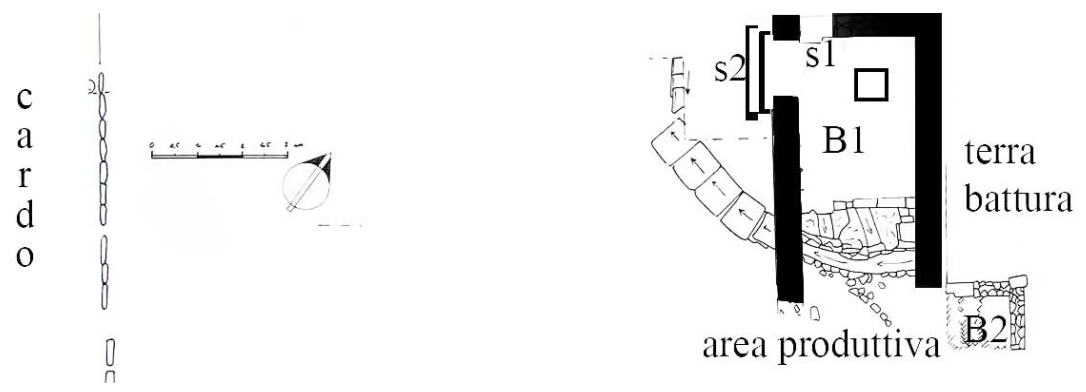

Fig. 13 - Edificio B: Fase I.

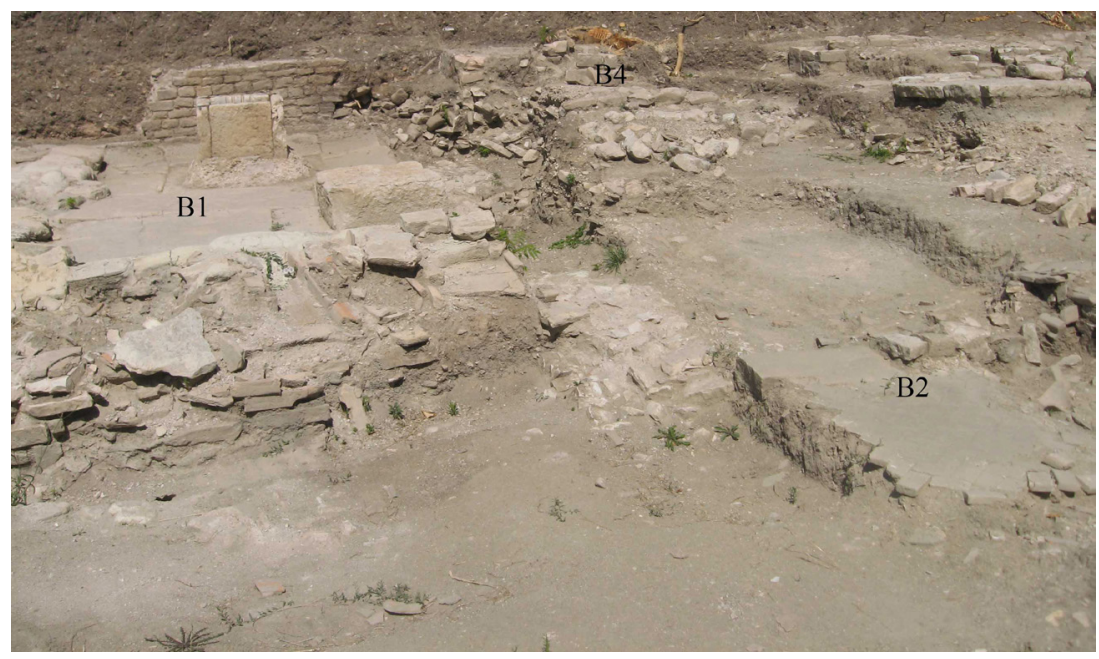

Fig. 14 - Edificio B: impluvium (B 1); area in opus spicatum (B 2); pavimento in cocciopesto (B 4).

\section{Fase $\mathrm{V}$}

In A 10 si realizza il canale orientale, che è chiaramente successivo, in quanto si collega all'altro sfondandolo. In questa stessa fase si chiude il passaggio da A 8 ad A 9 (Fig. 12) e quello da quest'ultima stanza ad A 10.

Elementi datanti: tecnica muraria. Nel chiudere le due porte si usano muri con alternanza di filari in pietra e laterizio (Fig. 12), questa tecnica compare a partire dal 62 d.C., ma è frequentemente impiegata fra II e III, soprattutto ad Ostia, esistono anche casi di IV ${ }^{12}$, a Forum Sempronii ricorre nelle fauces della "Domus del mosaico dei cani", lo stile dei mosaici di questo edificio e il ritrovamento entro il muri di uno dei due cubicula di una moneta di Severo Alessandro, che dato l'ottimo sta- 


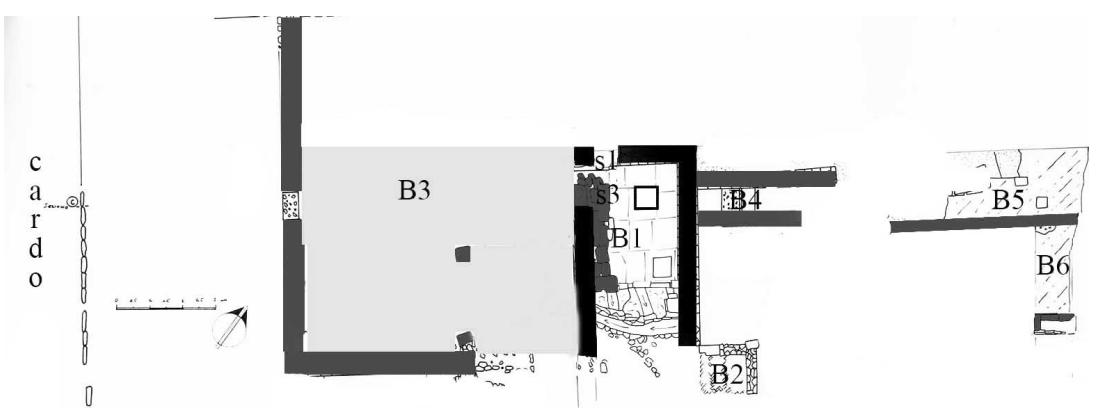

Fig. 15 - Edificio B: Fase II.

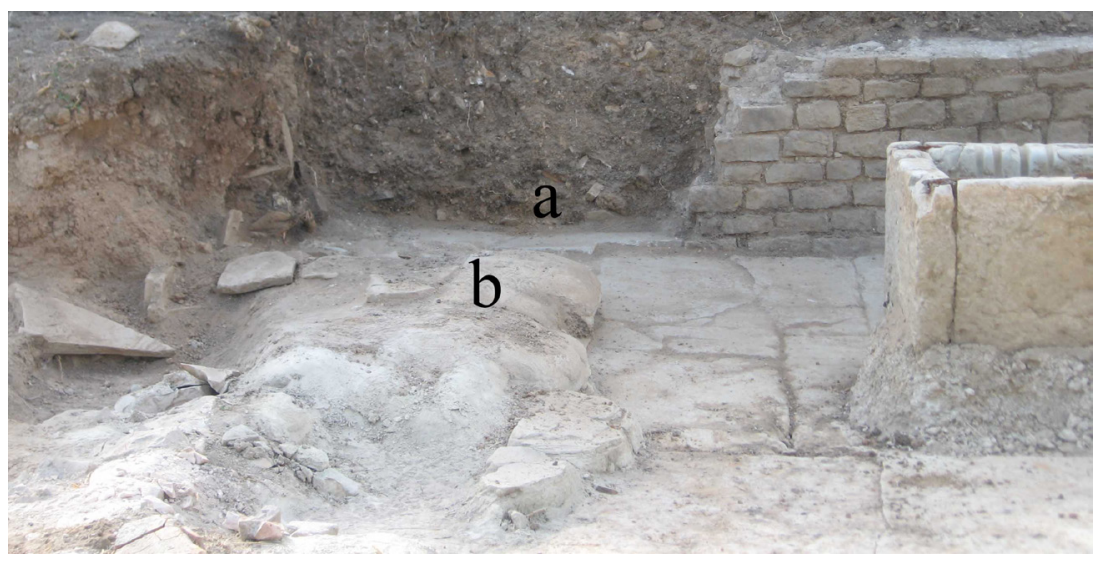

Fig. 16 - Edificio B, impluvium (B 1), le due soglie di epoche successive: (a) più antica (s1); (b) più recente realizzata con blocchi di arenaria reimpiegati (s3).

to di conservazione non dovette mai essere in circolazione ${ }^{13}$, possiamo ritenere che anche a Forum Sempronii questa tecnica fosse usata nel III d.C. Datazione: III d.C.

\section{Edificio B}

\section{Fase I (Fig. 13)}

Ledificio è caratterizzato dalla presenza di un impluvium (B 1) nel quale ci sono due soglie una a nord compresa fra due muri (s1), unaltra immediatamente a sud di quella sul versante ovest, realizzata con un blocco di arenaria ${ }^{14}$, chiaramente un riutilizzo e che quasi oblitera la prima soglia, dunque è più tarda.

13 Si tratta verosimilmente di un deposito di fondazione, sull'argomento: FACCHINETTI, 2012.

14 Questo materiale è tipico dell'epoca repubblicana a Forum Sempronii 


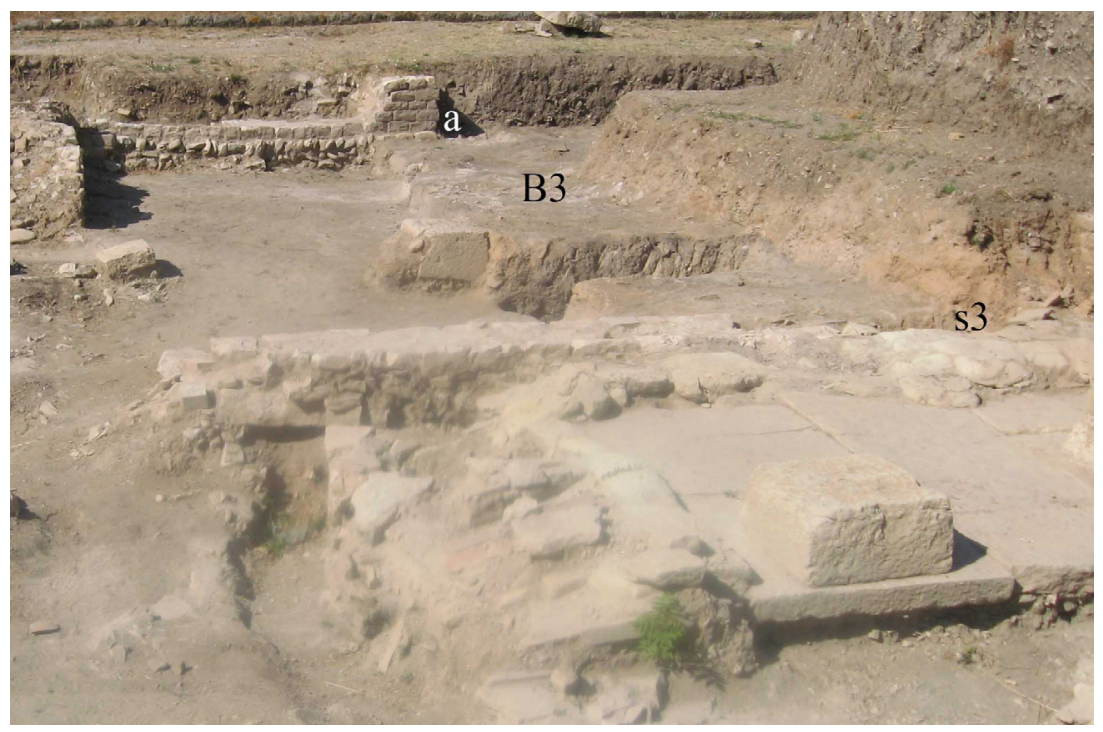

Fig. 17 - Edificio B, Fase II: (a) soglia di accesso da cardo; pavimento in battuto (B 2); soglia (s3).

Va tuttavia notato che il muro che delimita s1 a ovest non si lega con quello che dallo stesso lato delimita l'impluvium e come quello corre in senso nord-sud, quindi fra questi due muri doveva comunque esserci un passaggio (s 2), al quale forse si accedeva tramite uno o due gradini.

A est dell'impluvium c'era un'area con pavimentazione in terra battuta e frammenti ceramici, a sud della quale si trovano i resti di un pavimento in spicatum (B 2), che potrebbe essere connesso con questa prima fase, ma solo scavi futuri potranno accertare questo fatto (Fig. 14). Doveva trattarsi di unarea produttiva, che molto probabilmente restò in uso fino alla distruzione dell'edificio. Non è possibile accertare se a est dell'impluvium in questa prima fase ci fossero delle stanze. Elementi datanti: si veda Fase II; tecnica edilizia in opera vittata. Datazione: I d.C.

\section{Fase II (Fig. 15)}

In un secondo momento venne posta in opera la seconda soglia dell'impluvium (Fig. 16) e con essa altri blocchi di arenaria, lungo lo stesso lato. Questa soglia si connette con un pavimento in terra battuta (B 3), che va a legarsi ad un'altra soglia a ovest, così che si può pensare che in questa seconda fase si potesse accedere all'Edificio B, dal cardo che corre a occidente (Fig. 17). A Est dell'impluvium, venne realizzato un corridoio (B 4), pavimentato in cocciopesto, che correva in senso Est Ovest e si trova una quota più alta rispetto all'impluvium (Fig. 18; 19, a). Questo 


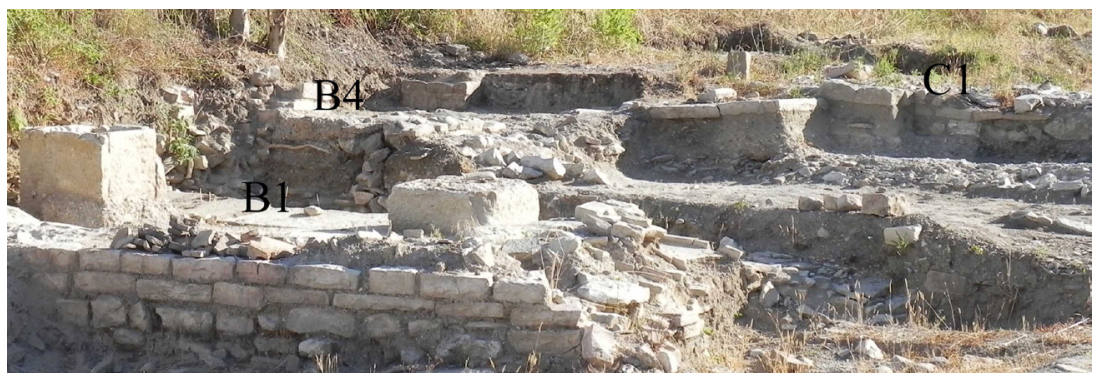

Fig. 18 - Edificio B: impluvium (B 1); corridoio con pavimento in coccio pesto della seconda fase (B 4); ultima fase (C 1).

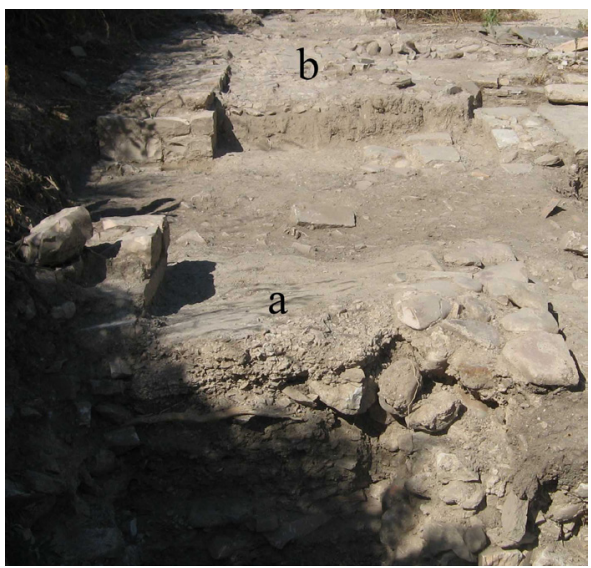

Fig. 19 - Edificio B: (a) pavimento in cocciopesto della Fase II; (b) pavimento dell'ultima fase, realizzato obliterando il precedente.

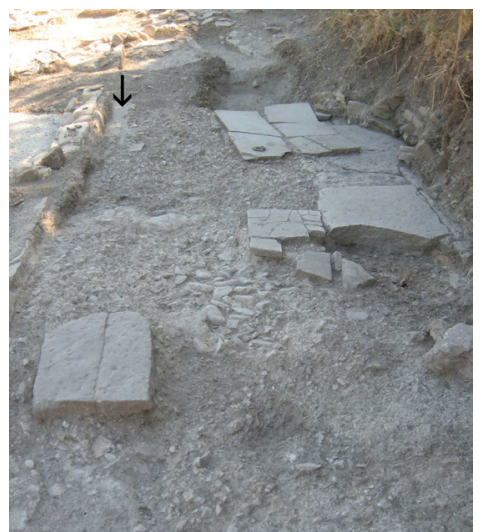

Fig. 20 - Edificio B: area con pavimentazione lapidea centrale e in cocciopesto ai lati (frustulo freccia nera). Forse area porticata.

corridoio probabilmente conduceva ad un area (B 5) che in buona parte giace sotto il limite di scavo, ma che da quanto è attualmente visibile era caratterizzata da una zona periferica in signunum e da una centrale in lastre lapidee, doveva trattarsi di unarea, che almeno in corrispondenza dei lastroni centrali doveva essere a cielo aperto (Fig. 20). Un elemento lapideo presente nel signimun, che potrebbe esser interpretato come basamento, indurrebbe a pensare che si trattasse di un'area porticata ai lati, fatto che potrà essere verificato solo con scavi futuri.

Questarea è a un livello più alto rispetto a quello del corridoio con pavimento in cocciopesto (B 4), il che potrebbe indurre a pensare che sia più tardo di quello, ma se si considera con attenzione il nuovo assetto che l'Edificio B ebbe in questa seconda fase, con ingresso a ovest, che conduceva all'impluvium, corridoio che da quest'ultimo si dipartiva, andando verso est, fino a giungere all'aera porticata, si coglierà il chiaro intento di volere uniformare l'edificio ai canoni dell'edilizia privata più 


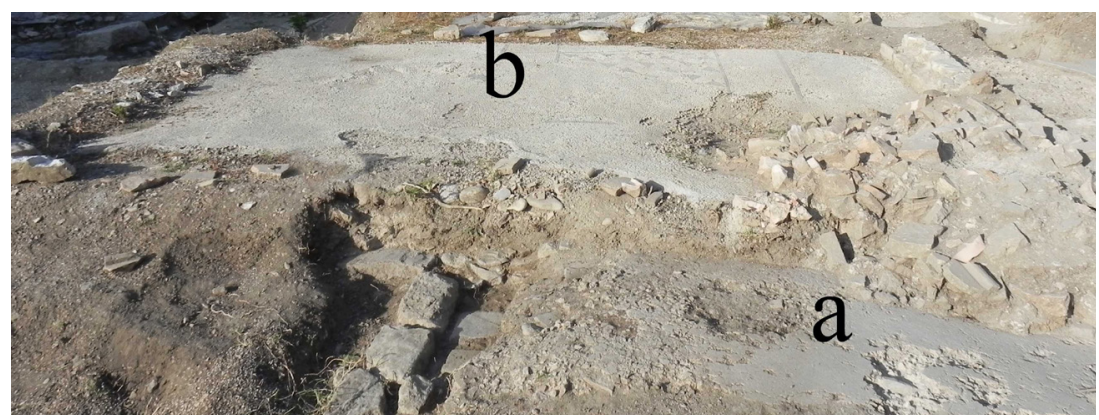

Fig. 21 - (a) pavimento in cocciopesto pertinente alla Fase II dell'edificio B; (b) pavimento musivo pertinente all'epoca dell'unione dei due edifici (AB 6).

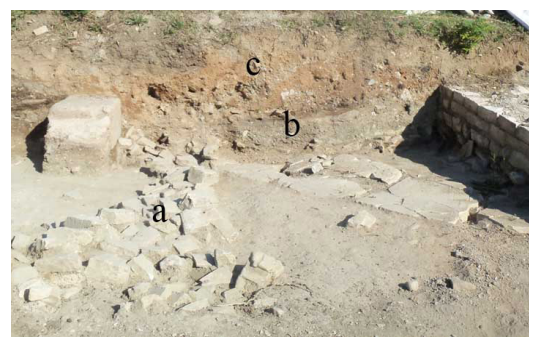

Fig. 22 - Edificio B: (a) "rudus" realizzato per spianare e impostare il pavimento in terra battuta B 3 (b); (c) crollo dei mattoni crudi avvenuto presumibilmente nel IV d.C.

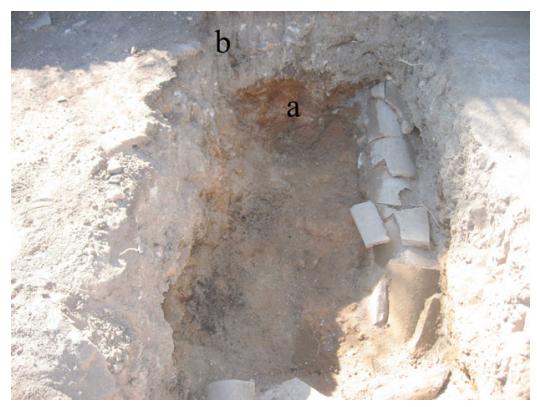

Fig. 23 - Edificio B: (a) mattoni crudi usati per spianare l'area per impostare B 3 (b).

prestigiosa, caratterizzata dalla successione: ingresso, impluvium, peristilio/cortile interno, questo sembrare parte di un progetto coerente farebbe ritenere l'area B 5 pertinente a questa seconda fase. Il dislivello potrebbe semplicemente essere dovuto alla necessità di adattarsi al terreno. A sud di B 5 c'era una stanza (B 6), delle cui dimensioni non possiamo essere certi, pavimentata in cocciopesto, successivamente obliterato da un mosaico (Fig. 21).

Questa seconda fase dovette esser conseguente ad un evento traumatico che causò la distruzione, se parziale o totale, non lo sappiamo, di un edificio più antico, si deduce questo dal reimpiego di blocchi di arenaria e anche dalla stratigrafia sottostante il pavimento in terra battuta che collega la soglia sul cardo all'impluvium. Fu inizialmente realizzato una sorta di "rudus" con pietre, frammenti ceramici e laterizi, sul quale furono posti i resti di mattoni in argilla cruda, sopra di questo secondo strato venne realizzato il pavimento in terra battuta (Figg. 22-23). $\mathrm{Nel}$ "rudus" e immediatamente al di sotto di quello sono stati trovati numerosi reperti compresi fra la tarda età repubblicana e quella augustea. Elementi datanti: tecnica muraria in opera vittata; monete datate fra monete datate fra 88 e il 4 a.C.; frammento di lastra campana databile 
fra I a.C. e I d.C. ${ }^{15}$, questi si trovano nel "rudus" del pavimento in terra battuta, quindi provano che questo è stato realizzato dopo l'inizio del I d.C. Datazione: I d.C.-II d.C.

\section{Fase III (Fig. 24)}

È questa la fase dell'accorpamento, nella quale il pavimento in cocciopesto in $B 6$ viene obliterato da un mosaico e si crea $A B$ 6, si creano poi i vani: $A B 1$ che doveva essere quello di passaggio e connessione fra i due edifici accorpati; AB 2 anche questo pavimentato a mosaico, probabilmente un vano di rappresentanza; $\mathrm{AB} 3$ con mosaico con emblema centrale, purtroppo lacunoso; $\mathrm{AB} 4$, con pavimento musivo geometrico e decorazione pittorica alle pareti, è stato possibile ricomporre dei frammenti trovati in crollo, ne è risultato un pannello con il mito di Perseo e Andromeda; AB 5 anche questo con un mosaico di ottima fattura; $A B 7$ di questo vano resta solo un lacerto di mosaico e la preparazione dello stesso, perché in una fase più tarda sarà obliterato da un altro pavimento, pertinente ad un corridoio (Figg 26-28). In questa terza fase si realizza anche la vasca $(\mathrm{V})$. Elementi datanti: come fase III dell'Edificio A. Datazione: $150-200$ d.C.

\section{Fase IV (Fig. 25)}

Un evento traumatico causa la distruzione di buona parte dell'edificio, a seguito della quale, le rovine vengono spianate (Fig. 26), creando un nuovo livello di calpestio, sorge così l'edificio $C$, che viene realizzato obliterando il vano AB 7 (Figg. 26-28) e il suo mosaico. Viene obliterato anche $\mathrm{B} 4$, da un pavimento probabilmente musivo, attraversato da una canaletta (Fig. 19, b; 29). In B 4 vengono poste lastre di marmo riutilizzate (Fig. 20). Elementi datanti: due monete che per dimensioni e peso, dovrebbero essere di IV d.C., molto danneggiate dal contatto con fuco sono state rinvenute sotto il crollo dei muri (US 367). Nello stesso strato è stato rinvenuto un frammento di coppa carenata classificata come forma Brecciaroli 3, la cui produzione non sembra cominciare prima della metà del III d.C. ${ }^{16}$. Nello strato che corrisponde al piano antropico dell'ultima fase, quella successiva alla distruzione (US 369), sono stanti rinvenuti alcuni frammenti di "Olle tipo Classe", databili fra V e VI d.C. ${ }^{17}$. Un frammento di una di queste olle è stato rivenuto anche nella vasca $(\mathrm{V})$, è dunque verosimile che sia rimasta in uso anche dopo la distruzione dell'Edificio B. Datazione: fine IV, inizio V secolo.

15 Nella lastra è ritratto un satiro, inginocchiato e trova un puntuale confronto le lastre rinvenute a Roma, località Pietra Papa, databili fra I a.C. e I d.C.: RIzzo, 1976.

16 Brecciaroli Taborelli, 1978; Stoppioni, 2008.

17 Gelichi, 1998; Ermeti, 2012, in particolare p. 365-369. 


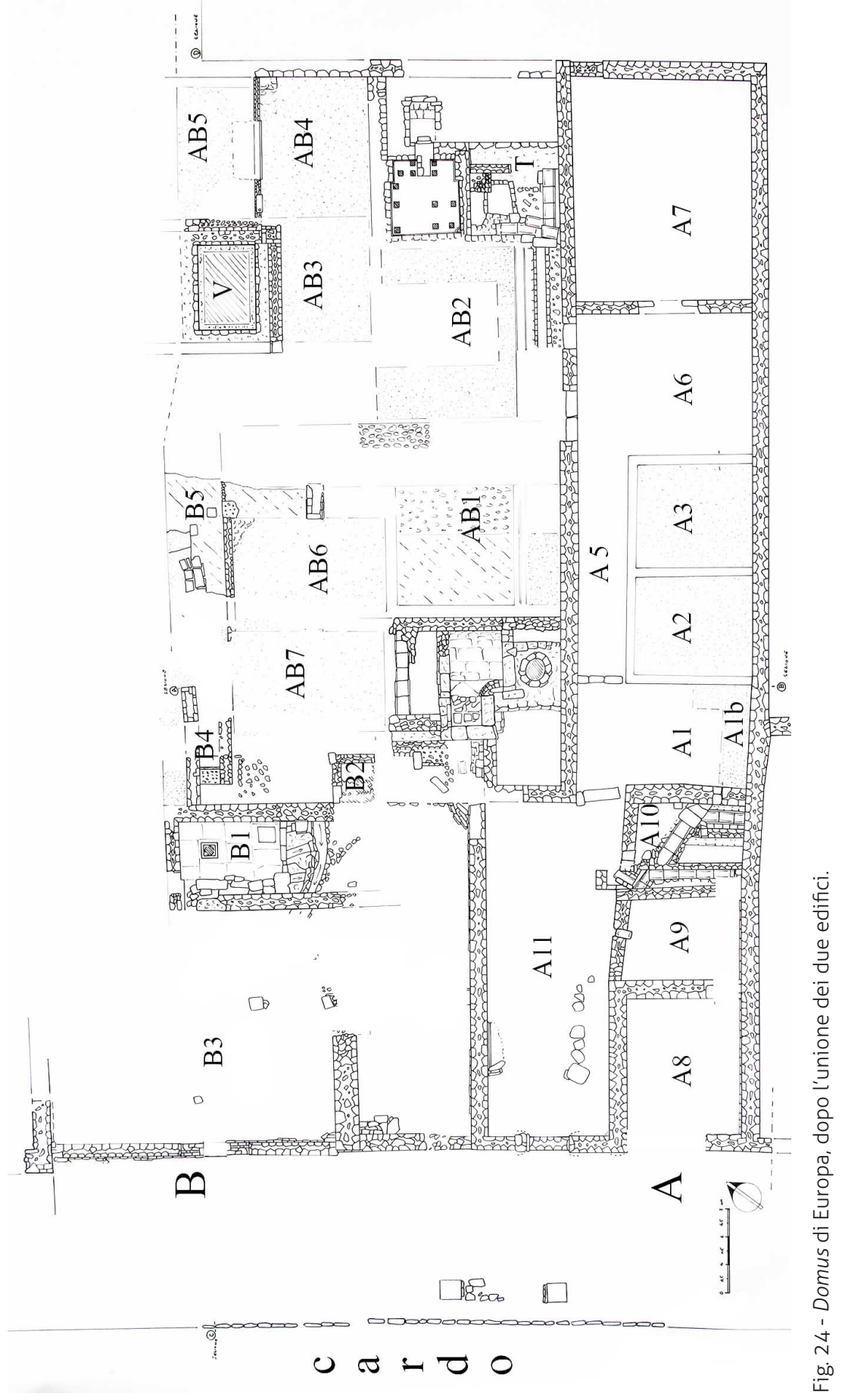



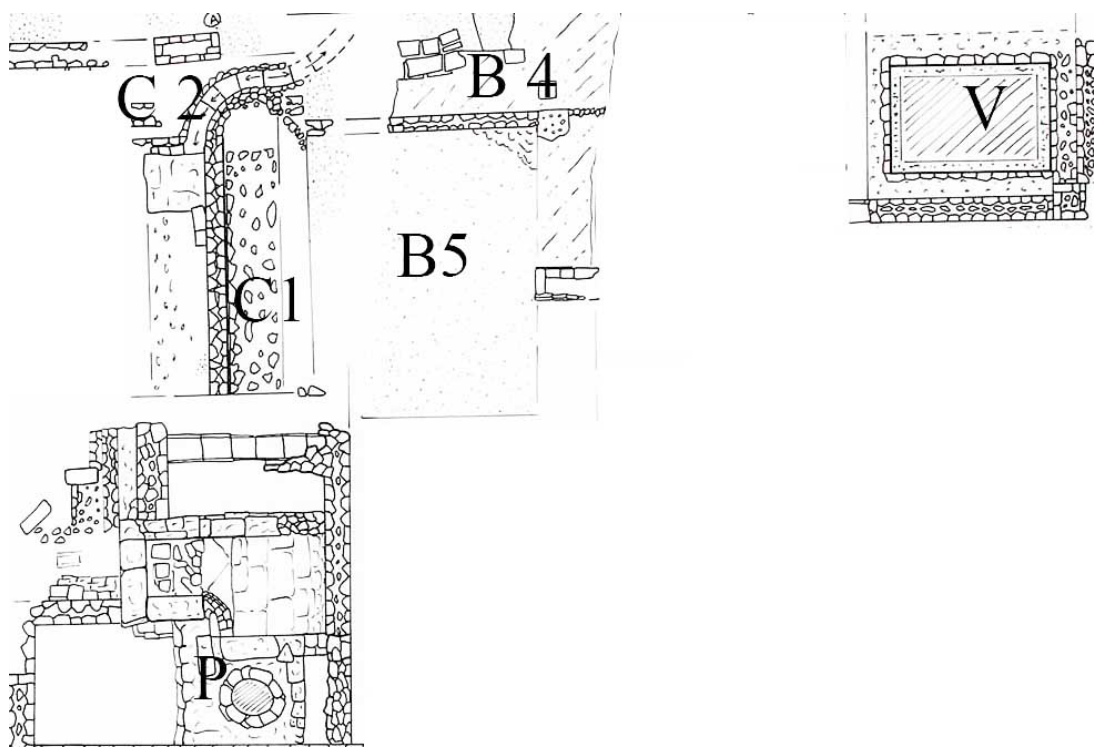

Fig. 25 - Pianta dell'edificio C.

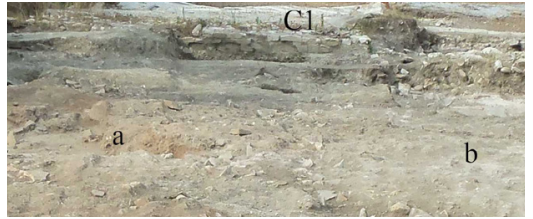

Fig. 26 - (a) crollo dell'elevato in mattoni crudi, spianato; (b) piano di calpestio, il tutto al livello dell'edificio $C$.

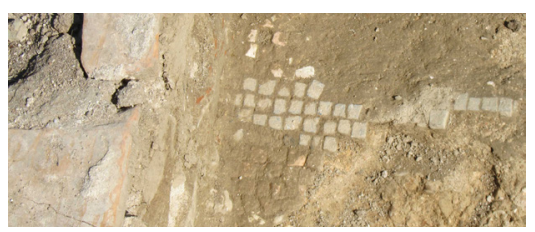

Fig. 28 - II muro dell'ambiente C 1 copre un mosaico d'epoca precedente.

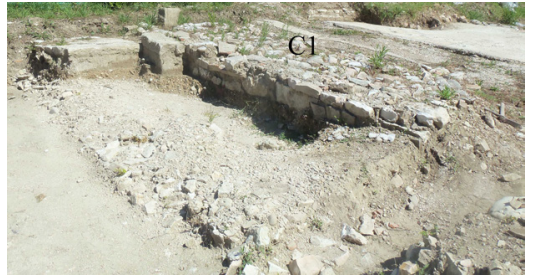

Fig. - 27 Edificio C: ambiente C 1.

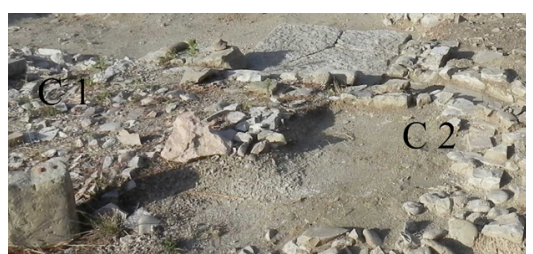

Fig. 29 - Edificio C: ambienti C 1 e C 2.

\section{LA DOMUS DI EUROPA FUNZIONI DEGLI AMBIENTI E RAPPORTO CON L'EDILIZIA PRIVATA ROMANA}

Per quanto concerne la planimetria sono già state poste in evidenza le analogie fra la pianta dell'Edificio A e le "casette tipo" ostiensi, ma notevole è anche la somiglianza con la Domus del Chirurgo di Rimi- 


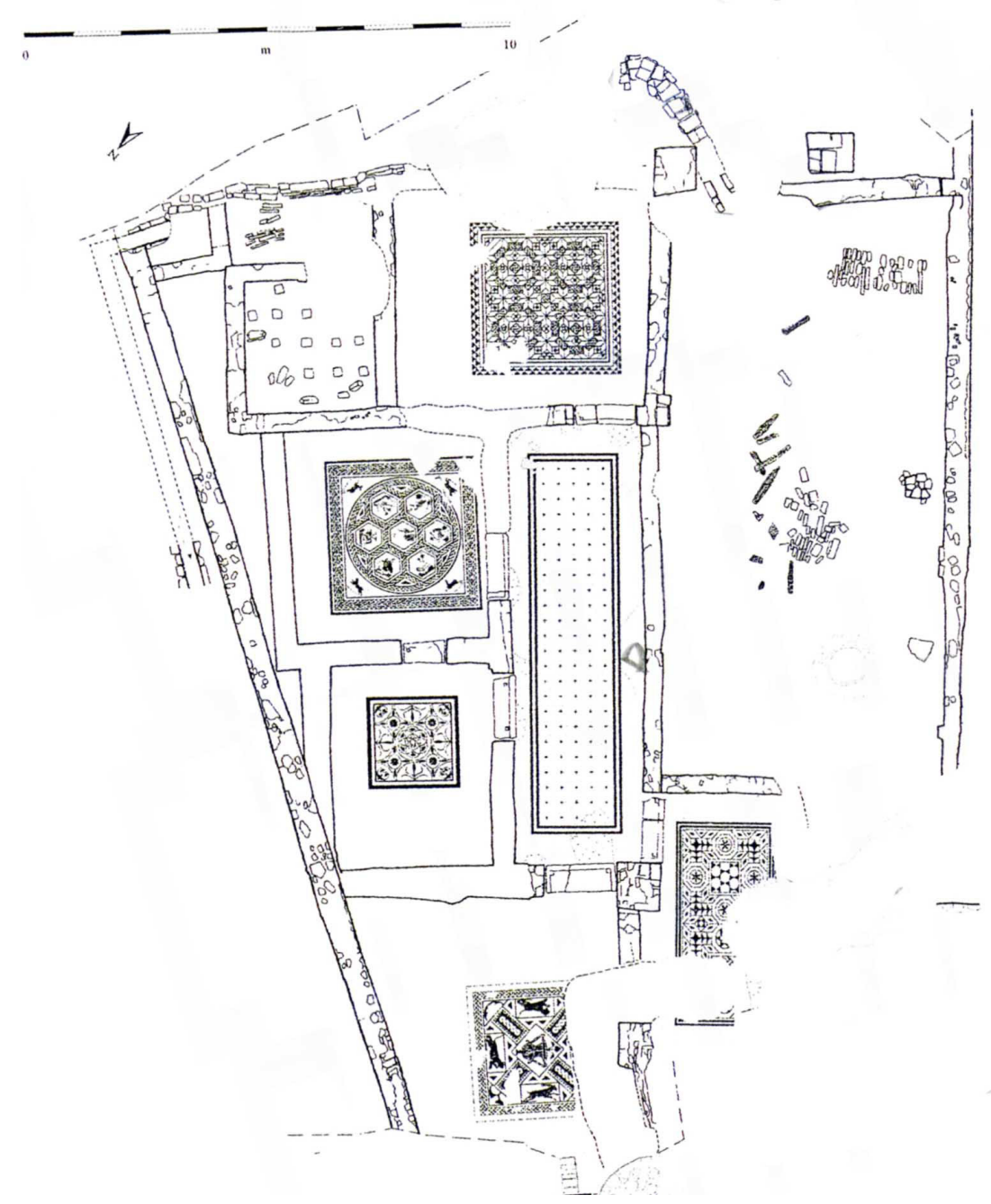

Fig. 30 - Ariminum, Domus del Chirurgo (GraZIANI, 2010, p. 58, fig. 13).

ni $^{18}$ (Fig. 30), qualche analogia esiste anche con un edificio noto come "Lotto B" di Libarna, in cui gli ambienti sono in fila lungo un lato di un corridoio ${ }^{19}$. Un esame complessivo dell'intera pianta della Domus di Europa rivela caratteri comuni con l'edilizia della Cisalpina, nella quale la planimetria delle case si distacca dai modelli canonici delledilizia privata romana, preferendo, al sistema fondato sullatrio, un impianto basantesi su corti e spazi aperti e corridoi di disimpegno, con una con-

18 Graziani, 2010, p. 31-42, 58-59; Ghedini, Annibaletto, 2012, p. 103-105.

19 Ghedini, Annibaletto, 2012, p. 316-317. 
seguente maggiore complessità dell'articolazione dei percorsi all'interno della casa, ove vengono a mancare i tradizionali rapporti di assialità e simmetria ${ }^{20}$. L'intero edificio manca di una progettazione coerente, ma è la risultante dell'adattamento alla conformazione del terreno e alla necessità di unire due strutture già esistenti, anche in questo caso, è possibile ravvisare unanalogia con l'ambito cisalpino ${ }^{21}$.

In alcune parti della Domus di Europa, tuttavia, si notano dei tentativi di riprodurre le forme planimetriche delledilizia privata centro italica: ingresso monumentale alla parte meridionale della domus (ex Edificio A), caratterizzato da una facciata che potremmo dire fastosa, con semicolonne, ma anche da un corridoio di accesso della larghezza di circa 6 metri e molto profondo, affiancato da stanze. È questo un chiaro tentativo di volere riprodurre un atrio tuscanico con alae. Un ingresso così ampio trova un confronto con la domus di Palazzo Massani ad Ariminum, ove l'ingresso assume la forma di un grande ambiente quadrangolare di $\mathrm{m} 4$ di larghezza ${ }^{22}$.

Nella direzione di un tentativo di riprodurre soluzioni planimetriche tipiche delledilizia privata centro italica potrebbe andare anche la successione ingresso, impluvium, corte/area porticata dell'Edificio B, ma come già detto questa potrebbe essere una mera suggestione, generata dal fatto, che la maggior parte di questa porzione dell'edificio non è stata scavata.

La parte coincidente con l'antico Edificio A dovette essere quella privata, ciò è deducibile dal fatto che nella soglia fra A 1 e A 5, realizzata all'epoca dell'accorpamento dei due edifici, sono presenti i fori per i cardini di una porta: una di quelle "barriere", quei diaframmi fisici con i quali si regolava l'accesso a quegli ambienti destinati ad un pubblico più selezionato: in genere i cubicula e $\mathrm{A} 2, \mathrm{~A} 3$ sono proprio stanze da letto, questo concetto di selettività sembra inoltre ribadito nel mosaico di Europa che pavimentava A $7^{23}$.

Le stanze AB 1-7 sembrerebbero invece connotarsi come ambienti di rappresentanza, non a caso l'accesso a questi, era possibile anche dall' Edificio B. Fra i vari ambienti con pavimentazione a mosaico di questa zona di particolare rilevanza è il vano $\mathrm{AB} 4$, caratterizzato da un pavimento musivo geometrico, nel quale però cè un'ampia area bian$\mathrm{ca}$, all'estremità orientale, chiaramente riservata ad un letto, l'ambiente per le sue dimensioni superiori a quelle dei cubicola A 3 e A 4, per la fastosità della decorazione parietale e per il fatto di essere preceduto, in perfetta assialità da un altro ambiente con pavimentazione a mosaico (AB 3), è possibile che fosse un cubiculum di apparato ${ }^{24}$. AB 6 avrebbe

20 Helg, 2012.

21 Helg, 2012.

22 HeLg, 2012, p. 40.

23 Venturini, 2007, p. 79-80.

24 Sull'argomento si veda BUENo, 2012. 


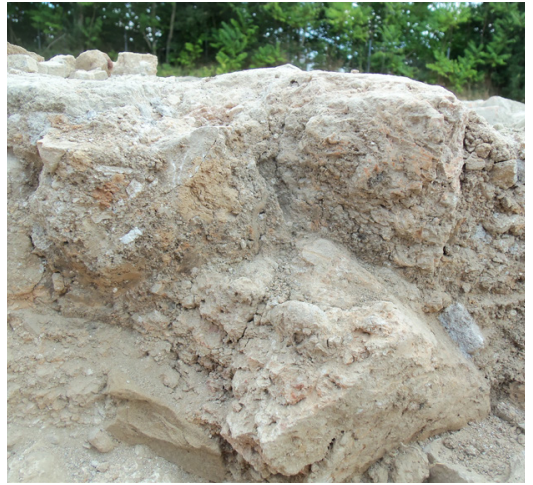

Fig. 31 Domus di Europa: mattoni di argilla cruda in crollo.

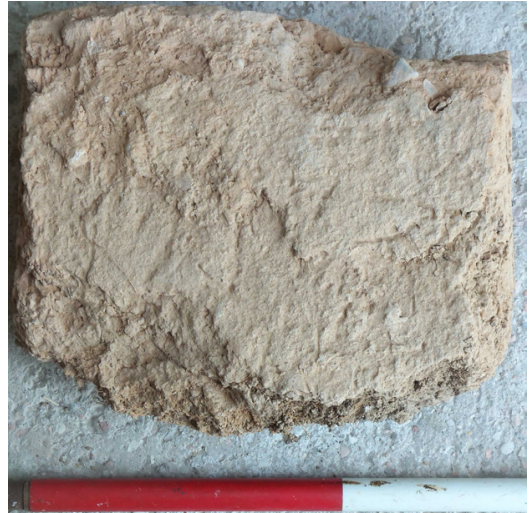

Fig. 32 - Un mattone in argilla cruda.

potuto svolgere la funzione di triclinium, un stanza per banchetti meno esclusiva di quella con il mosaico di Europa (A 7) e probabilmente AB 2 era il tablinum. AB 1 era verosimilmente un grande ambiente di smistamento, che segnava il limite fra la pars publica (AB) e quella privata (A).

La parte occidentale dell'Edificio B (B 1-3) sè supposto che avesse una funzione produttiva, non è certo di che tipo di attività si trattasse, ma va rilevato che qui sono state rinvenute alcune scorie di ferro, un'attività di questo tipo nel centro di una città, all'incrocio di due vie di importanza fondamentale e vicino al foro, potrebbe sembrare assurda, eppure in altri casi è attestata ${ }^{25}$. Nell'ambiente a ovest del pozzo $\mathrm{P}$ furono rinvenute quattro anfore segate nella parte sommitale ed interrate, questo ricorda il caso di Aquae Statiellae, ove c'era una casa laboratorio, per la lavorazione dell'argilla ${ }^{26}$. Per il momento si preferisce non esprimersi chiaramente sul tipo di lavorazione, ma è molto verosimile che questa fosse unarea adibita a funzioni produttive, il cui ingresso era indipendente rispetto a quello all'area degli ambienti A 1-7, anche questo aprentesi sul cardo, ma decisamente più dimesso.

Per quanto riguarda la tecnica edilizia riscontriamo l'uso dell'opus graticium per la divisione degli ambienti, mentre dallo scavo dell'Edificio $\mathrm{B}$ possiamo dedurre che i muri portanti fossero realizzati con uno zoccolo in pietra e un alzato in mattoni di argilla cruda (Figg. 31-32), tecnica che ricorre anche nella Domus del Chirurgo di Rimini, ma anche in alcuni edifici della Venetia et Histria e della stessa Regio VI, Umbria, come attestato a Sarsina ${ }^{27}$. 


\section{CONSIDERAZIONI FINALI}

Il presente contributo non è il sigillo di chiusura apposto allo studio della Domus di Europa, ma è una tappa, un momento di sosta in questo lungo cammino di ricerca, per fare il punto sulle acquisizioni, sui dubbi, sulle questioni ancora aperte o che si riaprono, presentandosi sotto una nuova luce, grazie ai nuovi dati raccolti.

La genesi e l'evoluzione planimetrica del così detto Edificio A possono rientrare fra le acquisizioni, così come l'epoca dell'unione con l'Edificio B e la nascita della domus, così come oggi la vediamo. L'Edificio $B$ è decisamente più problematico, soprattutto per il fatto di non essere completamente scavato, ma anche perché quanto di questo è stato riportato alla luce, lo è stato negli ultimi anni (2015-2017) e non tutto il materiale di scavo è stato completamente ed esaustivamente studiato. Di vitale importanza è stato avere avuto la possibilità di scavare il crollo dell'edificio, potendo così dedurre che fu distrutto da un evento traumatico e che questo probabilmente avvenne nel IV d.C. Estremamente rilevante è anche l'aver potuto constatare che sulle macerie della domus, $\mathrm{ma}$ anche in continuità con essa, sorse un nuovo edificio.

Il fatto che la domus abbia continuato a vivere fino al IV d.C. riapre anche la questione della datazione del mosaico di Europa, esteticamente attribuibile al IV d.C. ${ }^{28}$, ma fino ad oggi non esisteva alcun dato di scavo che consentisse di andare oltre la fine del II d.C., inizio del III d.C.

Un altro problema aperto è quello dell'attività produttiva che si sarebbe svolta nella domus, come sè visto ci sono indizi che instradano in varie direzioni.

Con sondaggi futuri si spera di potere anche fare luce sulla fase repubblicana-proto imperiale, intuibile da vari dati, ma al momento non chiaramente definibile.

Infine cè un problema che riguarda l'intero assetto urbano di Forum Sempronii. A ovest delledificio correva il cardo che portava al foro della città, attualmente la via si mostra pavimentata in lastre lapidee e si trova ad una quota più alta rispetto a molte delle stanze della domus e anche rispetto all'ingresso monumentale. Questo è dovuto al fatto che la via fu pavimentata dopo l'inizio del II d.C. e in quell'occasione fu anche innalzata di livello, tale pavimentazione fu anche loccasione per normalizzare la pendenza della strada.

Lepoca dell'accorpamento delle due unità abitative e della creazione della facciata monumentale dovrebbe essere compresa fra il 150 e il 200 a.C., visto che l'ingresso è ancora ad un livello più basso della strada lastricata, è logico pensare che il cardo allepoca corresse ancora ad una quota più bassa. Possiamo quindi ipotizzare che la lastricazione sia avvenuta dopo l'inizio del III d.C. Fra la domus e il cardo ceè un'area 
con una preparazione pavimentale piuttosto accurata ed alcuni blocchi che dovevano essere le basi di un colonnato, quest'area è al livello della strada lastricata, nella preparazione pavimentale è stata rinvenuta una moneta di Aureliano, che fu imperatore fra il 270 e il 275, si può dunque pensare che la pavimentazione e l'innalzamento di livello siano databili entro il III d.C., fatto ciò si pensò di realizzare un porticato che coprisse le facciate degli edifici e le loro irregolarità. Si dovrebbe così ipotizzare che la lastricazione e la sistemazione delle vie più importanti della città sarebbe avvenuta in epoca piuttosto avanzata, ma anche tale questione potrà essere risolta solo in futuro, anche con una migliore conoscenza proprio della Domus di Europa ed una più precisa definizione delle sue fasi strutturali. 


\section{BIBLIOGRAFIA}

ADAM, J.P. Larte di costruire presso i Romani, Milano, 1988.

Annibaletto, M. "Ambienti di servizio", in F. Ghedini, M. Annibaletto (eds.), Atria longa patescunt. Le forme dell'abitare nella Cisalpina romana (saggi), Roma, 2012, p. 135-155.

Brecciaroli Taborelli, L. "Contributo alla classificazione di una terra sigillata chiara italica”, Rivista di Studi Marchigiani, 1, 1978, pp. 1-38

Bueno, M. “Spazi riservati”, in F. Ghedini, M. Annibaletto (eds.), Atria longa patescunt. Le forme dellabitare nella Cisalpina romana (saggi), Roma, 2012, p. 97-110.

Bueno, M. Mosaici e pavimenti della Toscana, Padova 2011.

Cerato, I. "Strutture murarie", in F. Ghedini, M. Annibaletto (eds.), Atria longa patescunt. Le forme dell'abitare nella Cisalpina romana (saggi), Roma, 2012, p. 217-225.

Clarke, J.R. The houses of Roman Italy, 100 B.C.- A.D. 250: ritual, space, and decoration, Berkley, 1991.

ERmeti, A.L. "Forum Sempronii in età tardoantica e altomedievale. La documentazione materiale", in M. Luni, O. MeI (eds.), Forum Sempronii I, Urbino, 2012, p. 341-384.

Facchinetti, G. "Ritualità connesse alla costruzione di Domus. Le offerte monetali di fondazione in Aquileia", in J. Bonetto, M. SAlvadori (eds.), L'architettura privata ad Aquileia in età romana, atti Padova 21-22 febbraio 2011, Padova 2012, p, 337-351.

Falzone, S. (ed.). Scavi di Ostia XIV, le pitture delle insulae, Roma, 2004.

Gelichi, S. "Ceramiche 'tipo Classe" ”, in L. SAguì (ed.), Ceramica in Italia VIVII secolo. Studi in onore di J. Hayes, Firenze, 1998, p. 481-485.

Ghedini, F., Annibaletto, M. (eds.), Atria longa patescunt. Le forme dellabitare nella Cisalpina romana (saggi), Roma, 2012.

Graziani, G., Poloni, G. "Indagini su pitture parietali da contesti privati di Forum Sempronii”, in M. Luni, O. MeI (eds.), Forum Sempronii I, scavi e ricerche dal 1974 al 2012, Urbino, 2012, p. 171-194.

Graziani, S. "Abitare in città nella Romagna romana", in A. Coralini (ed.), Cultura abitativa nella Cisalpina romana 1. Forum Popili, Firenze, 2010, p. 25-100.

Helg, R. "Ingressi”, in F. Ghedini, M. Annibaletto (eds.), Atria longa patescunt. Le forme dellabitare nella Cisalpina romana (saggi), Roma, 2012, p. 37-43.

Ortalli, J. “Rimini. La Domus 'del Chirurgo' ”, in M. Marini Calvani (ed.), Aemilia, Bologna, 2000, p. 513-518.

Pavolini, C. La vita quotidiana a Ostia, Bari 1991.

Rinaldi, F. Mosaici e pavimenti del Veneto, Padova 2007.

Rizzo, M.A. "Su alcuni nuclei di lastre "Campana" di provenienza nota", Riv. Ist. Arch. Stor. Arte 23-24 (1976), p. 5-93.

Stoppioni, M.L., "La sigillata tarda di Sarsina", in A. Donati (ed.), Storia di Sarsina I. L'età antica, Cesena, 2008, p. 713-762.

Venturini, F. "I mosaici di Forum Sempronii", in M. Luni (ed.), Domus di Forum Sempronii, Roma, 2007, p. 53-90. 